\title{
ACCESS TO MATHEMATICAL OBJECTS
}

KEITH HOSSACK

Birkbeck College

London University

This paper questions the familiar doctrines that mathematical terms stand for objects and that mathematical proofs are logical deductions. It suggests instead that grasp of a mathematical concept typically requires mastery of an associated technique or procedure, and that many proofs rely upon our reflecting on how the relevant procedures would turn out. The proofs need worlds like our own spatio-temporal-causal one, and would fail at less obliging possible worlds: this implies that mathematical truths are not necessary since they do not hold throughout logical space.

\section{Introduction}

The mathematical objects theory is the doctrine that mathematics studies a special class of mathematical objects, just as physics studies physical objects, and biology living objects. On this view numbers and points literally exist and are just as real as electrons and protozoa. Mathematical facts are facts about mathematical things.

Deductivism is the doctrine that a mathematical proof is always a logical deduction. Deductivists recognize that in practice not all proofs are strictly valid, but demand that informal proofs must be completable as proper deductions if they are to count as proofs at all. Logicism, the doctrine that every math- 
ematical fact is a logical truth, is one amongst several species of deductivism.

Deductivism gives a special status to the axioms, which cannot be proved. One pressure towards the matematical objects theory is to suppose that the axioms are true because they report the facts about mathematical objects. We might instead try taking the axioms to be true by definition, but this is not a helpful move. As Mill ${ }^{1}$ pointed out, we can define as we please, but we cannot legislate for reality with our definitions to bring it about that anything in fact satisfies them. To insist that the axioms are true by definition is just to endorse "If-Thenism", the view held for example by Ayer ${ }^{2}$ that mathematics contains only the hypothetical truths that whatever satisfies the axioms satisfies the theorems too.

If-Thenism is open to Quine' $\mathrm{s}^{3}$ objection that there would then be nothing distinctive about mathematical truth. Any theøry whatever, about any subject matter you please, can be axiomatized in If-Thenist fashion. To distinguish mathematic from other sciences, we need to say what it is for the terms in the axioms to have a specifically mathematical interpretation. On theories of meaning inspired by the notion of a model for a formal language, the explanation can only be that their denoting

1 Mill [1967] Book II, Chap VI.2.

The proposition "A circle is a figure ... which has all its points equally distant from a point within it" is called the definition of the circle; but the proposition from which so many consequences follow ... is, that figures answering to this description exist.

2 Ayer [1967] chap. 4, p. 83.

All that the geometry itself tells us is that if anything can be brought under the definitions, it will also satisfy the theorems. It is therefore a purely logical system ...

3 Quine [1975] p. 83.

The body of all such statements ... is of course a part of logic; but the same is true of any "theory of deduction of sociology", "theory of deduction of Greek mythology", elc., which we might construct in parallel fashion with the aid of any set of postulates suited to sociology or Greek mythology. 
expressions are to be treated as names of the right sort of objects. In rejecting If-Thenism we can thus be led to the thought that there must be mathematical objects for the mathematical terms to stand for or denote.

The notorious difficulty is to say what mathematical objects can be. It would be absurd to say they are ordinary material objects: the number two is not a material object, nor is the point $A$ a fragment of matter. It would be equally absurd to say they are subjective mental entities: as Frege ${ }^{4}$ remarked, your number two would be different from everyone else's and all mathematical truths would be relative to individual persons.

And so we are driven to Platonism, the doctrine that mathematical objects belong to a third realm, neither physical nor subjective. We characterize abstract objects only negatively: they are not in space or time, and they do not interact causally with matter. How then do we know anything about them? Gödel's ${ }^{5}$ answer was that we have intellective access to them, trough mathematical intuition. But saying that the mind has special access to abstract objects seems to commit us to some species of dualism, since abstract objects could not interact with a material mind. Even if we have no objection to dualism as such, it remains obscure how the mind can contemplate objects directly, without the mediation of the senses.

An alternative is to see mathematical objects as theoretical posits with somewhat the same status as electrons have in physics. Since electrons and numbers are both needed in our best overall theory of the world, it is rational to believe in what our best theory needs. But the extent of the analogy between electrons and numbers is doubtful. For what makes a theory a

4 Frege [1968] section 27.

5 Gödel [1944] p. 456.

It seems to me that the assumption of such objects is quite as legitimate as the assumption of physical bodies and there is quite as much reason to believe in their existence. 
best theory is that it gives the best explanation of the course of events. To explain an event is to fit it into the causal pattern of the world. We need to posit electrons as the common cause of a host of statistically correlated events that would otherwise be unexplained. But if we are Platonists we do not think that numbers are the cause of anything. We can argue for electrons by inference to the best explanation, but causal inertness blocks the parallel argument for mathematical objects. The conception of mathematical objects as theoretical posits is thus no help to Platonism.

Perhaps we can have Platonism on the cheap, as in Crispin Wright's ${ }^{6}$ understanding of Frege. Or perhaps we need only pretend there are mathematical objects, as Hartry Field ${ }^{7}$ proposes in his doctrine of "fictionalism". But prima facie at least there is something to be said for exploring alternatives to the doctrine of mathematical objects.

\section{Logic}

We should also explore alternatives to deductivism. One obvious line of attack is to rely on Gödel's Incompleteness theorem, which shows that any theory adequate to express our mathematical knowledge cannot be effectively axiomatized, so that something must be amiss with the picture of mathematical truth as the logical consequences of axioms.

There is a general philosophical objection to deductivism that does not rely on Gödel. The chief advantage of deductivism is its apparent ability to explain unproblematically our epistemic access to some mathematical facts. But deductivism is only satisfactory as an account of our knowledge of theorems if Platonism is satisfactory as an account of our knowledge of axioms. If we are skeptical of the value of Platonism's account

6 Wright [1983].

7 Field [1989], Introduction. 
of the axioms, then we must be equally skeptical of deductivism's account of the theorems.

For what is a deduction? We might try to define it in terms of the logical consequence relation. ${ }^{8}$ Then someone makes a deduction from $P$ if they pass from $P$ to one of its logical consequences. We can say that $Q$ is a consequence of $P$ if every model of $P$ is a model of $Q$. But a model is a mathematical object, and so our knowledge of consequence relations seems to depend on our knowledge of the existence of the relevant mathematical objects. Our difficulties about knowledge of mathematical objects then carry over to corresponding doubts about our knowledge of the consequence relation.

We might instead seek to characterize consequence by relying on patterns of inference. But then the question arises how we know that the favoured patterns of inference are truth preserving. It will not do to appeal to the standard soundness proofs, since they rely on models and bring us back to the previous difficulty. We could try the linguistic move of appealing to the meanings of the logical connectives, and say for example that anyone who grasps the meaning of "and" can see that $P$ follows from $P$ and $Q$. Then $P$ logically implies $Q$ if there is a sequence of steps starting from $P$ that uses only such self-evident logical inferences and concludes to $Q$. But such an account is open to a fundamental objection of Wittgenstein's. ${ }^{9}$

A proof usually contains not one but many steps. If we define logical consequence in terms of a sequence of syntactically authorized transitions, we need to say how many transitions are allowed. If we make no restriction there will be sequences too long to be written down. If $Q$ is the conclusion from premiss $P$ of a sequence with an excessive number of steps, we encouter a dilemma when we enquire whether $Q$ is a logical consequence of $P$. Suppose we say it is. Then the existence of a sequence is

8 Tarski [1956] is an explicit statement of this account.

9 Wittgenstein [1967], Part II, 1--44. 
not the existence of any physical inscription of the sequence. So the sequence is not a material object, but some other kind of thing: in fact, it is just a mathematical object of the familiar sort. If we know of its existence it must be in the same way that we know of the existence of other mathematical objects. So once again deductivism would turn out to rely on mathematical objects for our access to mathematical fact.

The alternative is to say that the consequence relation holds only if there is an actual inscription of the sequence. But what we want logical consequence to capture is the impossibility of the premiss being true yet the conclusion false, and this surely cannot depend on whether there is an actual inscription of a sequence. Admittedly, some of the intuitionists have indeed held doctrines of this sort about proofs, arguing that mathematical facts are tensed. ${ }^{10}$ But it is difficult to see how the coming into existence of a proof can make it impossible for the conclusion to be false, if that was not impossible before.

Deductivism sees all proof as the tracing of logical consequence, and therein lies its mistake. The consequence relation belongs with the objective mathematical facts we seek to discover, whereas proof is an essential epistemic concept. If something does not lead us to knowledge then it simply cannot be proof. As Wittgenstein puts the point, a long "proof" is unsurveyable, and therefore not a proof at all. ${ }^{11}$

Call a sequence of logical inferences a canonical chain. Then $Q$ is a logical consequence of $P$ if a canonical chain connects $P$ and $Q$. Then it is possible to prove "If $P$ then $Q$ " by showing that there exists an appropriate canonical chain. The proof of existence can be short, for it need not exhibit the chain. Thus it is the short proof not the chain that is the real proof of "If $P$ then $Q$ ", because that is what gives us epistemic access to the mathematical facts. The short proof will in general not itself be

${ }^{10}$ For a discussion, see Dummett [1973], 6.1.

11 Wittgenstein [1967], e.g. Part II, 2. 
a canonical chain, so we conclude that there must be additional methods of proof beyond deduction in the strict sense.

\section{Diagrams and intuitions}

That deduction is not the only technique of proof is a contention familiar from intuitionism. And indeed it does seem plausible to look to intuition if we seek something other than logic to drive proof. The doctrine that intuition is the source of our mathematical knowledge derives from Kant. He says that to prove that $7+5=12$ we must give ourselves in intuition a case of $7+5$, which we then see is $12 .{ }^{12}$ Similarly in geometry we need to give ourselves a figure which we examine to confirm the required properties. ${ }^{13}$ Let us call the intuited object or objects the diagram. We can represent Kant as saying that we give ourselves a diagram in intuition, and that from an examination of it we see that things are as the theorem says.

What Kant says about geometry is sometimes disparaged because of the discovery of non-Euclidean geometry. But that affects only the axioms: what he says about geometrical proofs in Euclid's style surely remains correct. Visual inspection of a drawn or imagined diagram, intuition in Kant's terminology, is indeed needed in order to understand Euclid's proofs. Consider, for instance, the result that the angle in a semi-circle is a right angle. The proof begins with the diagram:

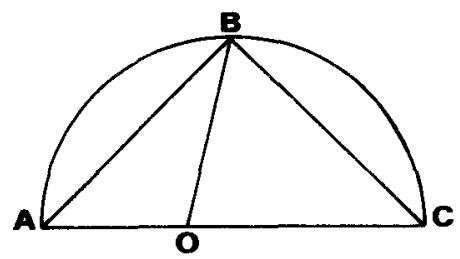

${ }^{12}$ Kant [1933], B15.

${ }^{13} \mathrm{Ibid.,} \mathrm{B} 65$. 
To understand the proof, it is necessary literally to see that the large triangle $A B C$ is composed of the two smaller triangles, and that the angle $A B C$ is made up of the two smaller angles. Thus the account that Kant gives has important strengths, for he has noticed a feature of classic mathematical reasoning that is overlooked by deductivism. But there are two problems for Kant, which arise also for the modern intuitionists who are his intellectual heirs.

The first problem is that if we have knowledge by inspection of the diagram in intuition, how are we supposed to tell that the inspection has been carried out correctly? Whence arises the required apodeictic certainty? Frege ${ }^{14}$ made fun of Kant by asking how we are supposed to know that J35664 + $37863=$ 173527 by inspection of the $a$ priori intuition, but the same question arises even in the case of $7+5=12$. The geometrical case is even more telling against Kant. The diagram may look as if it conforms to the theorem, but how are we to rule out the kind of very slight discrepancy that shows up not on visual inspection but only by measurement? Thus Kant cannot tell us how we can be certain of what we seem to see in the diagram.

The second problem is that the diagrani is just one case. How can we generalize from it to other cases? How can I tell that because this collection of $7+5$ is 12 , that every other collection of $7+5$ is 12 too? If this particular triangle in the diagram verifies some geometrical proposition, will every other triangle do so too? Kant relies on the Transcendental Aesthetic, but that is unlikely to satisfy the non-Kantian. If we are to make further progress, we need an alternative to the mathematical objects theory. 


\section{Procedures}

Wittgenstein sketches a different theory at the start of the Investigations. ${ }^{15}$ There he was concerned to attack "the Augustinian conception" of language, according to which words function essentially as names. A name gets meaning by standing for an object, so that understanding the name is just a matter of knowing which object it is that the name stands for. Against this, Wittgenstein considers someone going into a shop and asking for five red apples. To call "red" here the name of a colour is, he says, to obscure an important complexity in the word's use. The shopkeeper perhaps produces a colour chart and compares the appies with the sample marked "red": they are red if thcy match. According to Wittgenstein we might say the sample is part of the language, but neither it nor anything else is the designation of the name "red".

It is the same, he says, with the word "five". Again we have to take into account the full complexity of the linguistic practice. Before one can use number words, one has to memorize the numerals in the correct order. To give five apples, one has to master the technique of counting out. So to understand the meaning of "five apples", one needs to be master of the appropriate procedure, rather than needing to know of the mathematical object 5 .

This doctrine of Wittgenstein's can be applied to some other branches of mathematics. Just as arithmetic requires the procedure of counting, geomctry too has its procedures, the constructions. To understand what is meant by saying that something occupies a certain point, one might suppose that what is needed is to know which object the point is. But on the procedure account, there is no such object to know of, but only procedures of measuring. It is in the context of mastery of such procedures that one understands what it means to talk of geometrical locations.

Wittgenstein [1958] I.1. 


\section{Diagrams and certainty}

The observation that arithmetic and geometry both depend on procedures suggests how to repair the Kantian account of proof. The first problem was how we can be certain of what we see in say, the diagram for a proof, that $7+5=12$. On the procedure theory, we resolve the difficulty by simply inverting the argument. Being 12 just is being disposed to give the result " 12 " when counted by a suitably placed and competent person. The outcome of the person's application of the procedure is constitutive of what it is to have the mathematical property of being a collection of 12 . In the same way, being $7+5$ just is to be disposed to be counted as a 7 and a 5 by a suitably trained person who is in a favourable position to count.

Here we might press the analogy with the colour word "red". What it is to be red is to be disposed to appear red to a suitably placed observer, and the judgments of such observers are constitutive of what it is to have the property of redness. Predicates like "red" and " 12 " are markedly different from predicates which stand for what we might call intrinsic properties of things, the structural properties that give rise to dispositions like redness. Whether a thing has an intrinsic property depends on how it is with the thing as it is in itself, and cannot be definitely settled by simple visual inspection. Thus gold looks golden, but a golden appearance can be caused by properties other than being gold: being fool's gold is just as efficacious in this respect. So visual inspection under favourable circumstances is not usually sufficient to settle whether a metal is gold, since that is an intrinsic property. But visual inspection does settle the question of whether something is red. If something appears red in the right circumstances, that is constitutive of its being red.

There is then no sense to the suggestion that something might really be another colour even though it looks red under optimum viewing conditions. We can say the same about mathemat- 
ical predicates where there is a procedure that decides whether the predicate applies. It is not an intrinsic property of the objects that they are 12 , though it is their intrinsic properties that dispose them to count as 12 . It is constitutive of being 12 that they count as 12 , and there can be no question of their perhaps not being 12 if that is the count under optimum counting conditions. This point is overlooked both in Platonism and by Kant. Platonism with its doctrine of independent mathematical objects naturally takes numerical facts to be about intrinsic properties of the numbers, quite independently of any human counting activities. Kant too seems to take numerical properties of things to be intrinsic properties, so that the question will arise whether our intuition of these properties is reliable. And the answer to that must be, as Frege's mockery shows, that our intuition is completly unreliable. Intuitionism cannot explain the certainty of mathematical knowledge.

The procedure theory in contrast is well placed to explain the certainty. It says we are right to be certain of what we see in the diagram, because the diagram is just the sort of example that would be used in teaching someone to count. The possibility of certainty in cases like this is an essential precondition of the meaningfulness of the mathematical terms used in the theorem, for if there is to be a procedure of counting, it must be possible for us sometimes to know that we have counted correctly. If we doubt that that there are 12 things in this case, then we are doubting that we can give the sign " 12 " its usual meaning, and so the doubt is to that extent self-defeating. Thus the procedure account is not open to Frege's objection. It can concede a distinction between the cases of small and large numbers, and indeed can agree with Frege that proof of the correct way to add large numbers is not available along the lines of Kant's diagram. Very large numbers are not the paradigms we use in teaching counting. The procedure theory would say the proofs are quite different if the numbers are large, and that they rely on our grasp of further procedures such as the technique of 
counting in the decimal system. But that would not detract in the least from the status of the $7+5=12$ diagram as a proof.

\section{Generalizing from the diagram}

The second problem for a Kantian account is how we are to generalize what the one diagram shows us to other cases. One way to pass from the particular to the general is induction as in science, but that seems inappropriate for mathematical proof. Another way is to reason with an arbitrary object. We reason about the diagram, says Berkeley, ${ }^{16}$ not noticing its particular features but only those relevant to the proof. Then any other diagram agreeing with the first in all relevant features will fall under the theorem too, even if it does not share othcr features irrelevant to the reasoning. But this account, while it works well enough in the context of Berkeley's own theory, is surely not available to Kant, since by "reasoning" Berkeley can mean only deduction. If we do not rely on deduction, but appeal also to intuition of the properties of the diagram before us, then we cannot claim to treat it as an arbitrary object.

So if we wish to accept the role of diagrams in proofs, we need some third way to derive generality from a single instance, a way neither inductive nor deductive. There is such a way: the use of a particular diagram to represent a general method of solution of a practical problem. For example, if I am puzzling how to fit these parts together to make that shape, you can give me a diagram that shows me how to do it. The diagram is a concrete particular, but I use it to grasp a general method for putting together parts like this to form a shape like that. We get the result Berkeley aimed at. The diagram shows the method whether its parts are black or white, ink or chalk, large or small.

16 Berkeley [1710] Introduction XII.

He draws, for instance, a black line of an inch in length: this, which in itself is a particular line, is nevertheless with regard to its signification general... : so that what is demonstrated of $i t$, is demonstrated of all lines... 
The details of the diagram are unimportant, because we are concerned not with the diagram as object, but with the general method we grasp from it.

Let us return to Kant's proof of $7+5=12$. We are shown a particular case that is an instance of the theorem. The sight of the particular case, the diagram, is supposed to lead to knowledge that the theorem holds generally. We can perhaps improve on Kant's account if we say the generality arises because the diagram shows a general method of exhibiting a group of $7+5$ objects as a group of 12 objects. The method shown here is not very interesting, so we should perhaps think of this as a degenerate case. The account is more plausible when the method displayed involves a modicum of ingenuity, as in the case of the proof of the angle in the semicircle. The diagram shows how to decompose the angle, by showing us a particular case in which it has been done.

On the procedure theory, the picture of how mathematical proof often works would be as follows. Say we are trying to prove that all $A$ are $B$. Associated with the predicate $A$ will be a procedure or procedures which determine whether $A$ applies in a particular case. Similarly there will be procedures associated with $B$. What the proof does is to exhibit a particular diagram with two purposes to serve. First, it either is or represents something which is both an $A$ and a $B$, since we are able to see on inspection the outcomes of the relevant procedures. Secondly, we see from the diagram a general method by which to exhibit any $A$ as a $B$-for example, we see how to exhibit any angle in a semicircle as containing two isosceles triangles. Deduction and logical reasoning will have a role to play in reaching the final conclusion, but the diagram is needed too.

On this account it is absolutely characteristic of mathematical proofs that they exhibit methods. Our epistemic access to mathematical fact is best compared not with intuitions of objects, but with the acquisition of a praetical skill. 


\section{The problem of generality}

That rigorous proofs should make essential use of practical methods is obviously incompatible with deductivism. Neither can it be reconciled with the mathematical objects theory. Take a proof that shows that a certain kind of shape can be decomposed into four equal parts. What the proof shows is a method that actually accomplishes the decomposition, a method that would work quite well in practice on any suitable material of the right shape. A Platonist will wonder what this practical technique can have to do with facts of pure mathematics. Shapes are abstract objects, the Platonist says, and facts about our sublunary cuttings and pastings cannot reveal the properties of the abstract objects. But this conflict between the procedure theory and Platonism need not dismay the procedure theorist. On the contrary: the question of how mathematical facts find useful application is a notorious difficulty for the Platonist, whereas the problem does not even arise for the procedure theory. What is more, the procedure theory can account easily for the generality of the conclusion, whereas generality presents a fresh difficulty for the Platonist.

In discussing propositions containing a generality operator such as "all" or "every", it is helpful to make a distinction between a referential use where particular objects enter into the truth conditions, and an irreferential use, where this is not the case. For example, in "All Jack's sisters are blonde" we economically state a proposition equivalent to the conjunction of propositions saying of each that she has blonde hair. In contrast, "All swans are white" is presumably an irreferential use: here it is not conjunction that we want but quantifiers, which following Frege $^{17}$ and D.M.

\section{Frege [1891] p. 38.}

Such a function (sc quantification) is obviously a fundamentally different one ... for. .. only a function can occur as its argument... functions whose arguments are and must be functions... I call. .. second-level. 
Armstrong ${ }^{18}$ I take to indicate second-level properties of properties. The intention here is not normally to state economically a conjunction about individual objects but rather to connect the properties of being a swan and whiteness. In this case the connexion would presumably be thought of as a nomic one. Similarly "All unmarried men are men" is unlikely to be a referential use. The speaker means not to talk about the individual men but to state a connexion between being an unmarried man and being a man. This time the connexion would be a logical one.

The Platonist way to read a mathematical theorem is as telling us the relations in which the mathematical objects stand. We have already rehearsed the difficulties that beset Platonism in saying how we can have knowledge of individual abstract objects. Because it reads general statements in mathematics as referential uses about objects, Platonism faces a fresh puzzle about how we can know general theorems.

If the variables of quantification range over totalities which cannot be inspected in a finite time, there are two ways a finite creature can find out general facts. It can discover that a general proposition is false by recognizing a counterexample, and it can discover it is true by recognizing a finite proof. But if a proposition is known by recognizing a proof of it, the question in turn arises of how the premisses of the proof are known. It seems not to help the Platonist to say that the knowledge of the theorems rests ultimately on knowledge of the axioms, for if they are themselves general propositions it would appear their truth value cannot be known in either of the two ways a finite creature can know such things. If an axiom is true we shall not find its truth value by recognizing a counterexample, and if it is independent of the other axioms we shall not be able to prove it by a finite proof taking them as premisses. So it is hard to

18 Armstrong [1983]. Chapter 6 expounds his doctrine of laws of nature as "relations between universals". 
see how the axioms can be known, and if the axioms cannot be known the theorems cannot be known either.

Perhaps we could here make a move like Gödel's ${ }^{19}$ and argue that we are justified in believing the axioms not because they are self evident or something of that sort, but because they lead to fruitful consequences in the finitary domain. This has some plausibility if we are considering how a highly infinitistic theory like set theory might systematize modestly infinitistic theories like arithmetic. But it is unclear in what sense the finitary facts of computation stand in need of systematization or explanation. Moreover, as the finitary facts are all decidable the infinitistic theory yields no new finitary theorems. It is quite obscure how facts about the results of particular finite computations would jusiify believing general propositions. Consider the proposition that every number has a successor, so that there is no last number. We encounter quite large numbers, so we suppose that the number of numbers must be quite large too -but why must it be infinite, when the supposition that it was large but finite would fit the finitary facts just as well?

The alternative to the Platonist account is to read the general statement as irreferential, so that the objects drop out of its truth conditions. This removes the puzzle about how we can be acquainted with the whole of the infinite extension. Of course, it remains to give an account without appealing to extensions of what it is for there to be a connexion between properties. In the case of nomic connexion of properties, we can say that we are dealing with laws, to which we gain epistemic access by normal scientific inductive practice; in the case of logical connexion we gain access by deduction. Finally, in the mathematical case,

19 Gödel [1947] p. 477."

There might exist axioms so abundant in their verifiable consequences, shedding so much light upon a whole field, and yielding such powerful methods for solving problems... that, no matter whether or not they are intrinsically necessary, they would have to be accepted at least in the same sense as any well-established physical theory. 
the procedure theory says that general propositions are made true by facts about procedures. Thus Goldbach's conjecture is true, if it is, because the procedure of searching for an even number not the sum of two primes will never terminate. If a computer is programmed to carry out the search, then given that the machine has a deterministic construction, it is determinate whether it would halt, though this is not something that we can necessarily discover just by specifying the construction. The procedure theory avoids the intuitionist objection to the determinacy of truth value of undecided propositions by grounding them in facts about how the computer is going to behave. These facts are determinate because of the laws governing the construction of the machine, and the appeal to laws replaces the Platonist appeal to an actual infinite totality. Thus the procedure theory would sustain the law of the excluded middle, contrary to intuitionism. Of course it remains true on the procedure theory that we do not always know what outcome. is in fact determined by the laws, so that the procedure theory is not immune to general anti-realist objections of the sort raised by Michael Dummett. ${ }^{20} \mathrm{But}$ it is distinguished amongst realist theories by its parsimony. It replaces the infinite totalities of Platonism with the laws of nature as the objective ground for the determinateness of truth value of infinitary propositions.

\section{Methods and facts}

On the procedure theory, the proof shows a method such that given an $A$, I can exhibit it as a $B$. So the mathematical connexion between the properties $A$ and $B$ is shown by a method for exhibiting each $A$ as a $B$. I follow the proof and grasp from it a method of performing a certain task. Perhaps I form a mental picture of myself carrying out the task in the manner the proof indicates. This is my epistemic aecess to the method, and hence to the mathematical facts. 
I may believe in the existence of a method but be mistaken. When I put my supposed ability to the test I need the cooperation of reality if I am to get the results I want. This shows that to assert the existence of procedures and methods is to make factual claims about the world. The procedures are needed to give the mathematical terms a meaning at all; the methods are needed to exhibit the connexions of properties asserted in the theorems. In both cases we need cooperation from the world if mathematics is to succeed.

We need to consider whether mathematics can be done in a possible world, if the needed procedures cannot be carried out there. A preliminary point is that even if the procedure cannot be performed at a world, it can still be referred to there. The ability to count, if I have it, indeed depends on the world's cooperation, but I can still speak of counting even at an inhospitable world where counting is impracticable for me. All that is needed is that I should be able to fix the reference of the word "counting" by demonstrating the technique at a suitable tractable world - the actual world, for example.

The existence of a procedure is a precondition of mathematics at a world, but a procedure can exist in this sense even if it cannot reliably be put into effect. It may be that at a particular world, I cannot perform the procedure for unimportant reasons. For example, objects may move too fast for me ever to count them, given my powers at that world. That presents practical difficulties for arithmetic there. Or a world may happen to contain only liquids, so that there is no straight edge available for geometrical constructions. This led Russell ${ }^{21}$ to speculate about what progress the liquid geometers would make. But such practical snags do not prevent mathematical description of the state of affairs, provided we can say what the result of the procedure would have been had it not been for the difficulties. Thus mathematicians say their subject involves "ideal" operations:

Russell [1956] section 72. 
they like to talk of lines with length and no breadth, of inextensible strings, and of Turing machines with infinitely long tapes. Surely mathematicians are not serious about impossible objects like these? The explanation is that in practice procedures are disrupted by the finite breadth or extensibility or length of the tape, but that we can see clearly enough how things would have gone on if the procedures had not been disrupted. If the laws of nature are such as to make definite what would have happened without the disruptions, it is legitimate to talk of infinite tapes and the rest. Mathematical idealization is not the breathless positing of abstract ideal entities, but mundane recognition of counterfactual definiteness under the laws.

If we travel far in logical space from the actual world, we reach unfriendly worlds where substance is not conserved and causality fails. According to the procedure theory, mathematical description of such worlds is pointless, for we cannot perform the procedures and we cannot say either what the result of a procedure would have been if we could have carried it out. Consider a world where things sometimes appear or disappear of themselves without any law. Then the empirical results of counting would be quite unpredictable, and we could not say that given objects were disposed to elicit any particular numeral when counted. There would be nothing to support counterfactuals about what the result of a count should have been, and so the notion of correct counting would be empty. Thus counting depends on the world having an appropriate causal structure.

The same applies to geometry. In a causally anomalous world a Euclidean construction cannot be carried out with any confidence. If we add construction lines to a figure, we in the actual world believe that the rest of the figure will be unchanged unless some causal agent intervenes. So at the actual world, the following is true: if shapes or sizes are altered after a construction, then a causal explanation can be found. Again, in Russell's liquid world no constructions can be performed in 
practice. But geometry can still apply if the following is true: if it had been possible to carry out constructions, then there would have been a causal explanation of any alteration of shapes or sizes after a construction. But when causality fails the counterfactuals lapse, and the geometrical concepts too become empty.

Thus the procedures on which the mathematical terms depend for their sense may not exist at some possible worlds, which accordingly cannot be described in mathematical terms. Similarly the methods appealed to in a proof may not exist at a world. The statement that a method exists again entails counterfactuals about what would happen if I made no mistake and was not impeded. Thus the existence of a method at a possible world implies that the world occupies that subspace of logical space where the appropriate counterfactuals are true. Since there are possible worlds where the counterfactuals are false, it follows that mathematical theorems based on the existence of a method are false at some worlds, and hence are not logical truths.

The mathematically possible worlds are those where the propositions of mathematics are true. These worlds will all be such as to allow us to be confident of the effectiveness of the methods to which we appeal in our proofs. At other worlds, the propositions of mathematics are false, though not necessarily in the sense that some mathematical contrary of them is true. Thus there are worlds where it is not true that $2+2=4$, but it need not follow that at some world $2+2$ has some other definite value. $2+2=4$ will be false at a world at which the counterfactuals it supports are false, but it by no means follows that the counterfactuals supported by some mathematical contrary of it are true at that world. Thus the mathematical negation of a theorem, being itself a mathematical proposition, is true only under the assumption of counterfactual definiteness. The mathematical negation is therefore an internal negation and not the same as the regation of classical logic, and to that extent the intuitionist suspicion of the law of excluded middle is justified, 
though this is not grounds to suppose that classical logic itself needs revision.

\section{Is mathematics a priori?}

On the one hand the procedure theory says that the truths of mathematics are objective because laws of nature make it determinate what the outcome of a procedure should be. On the other hand, it stresses the role of proof in knowing mathematical theorems, suggesting that mathematical truth is discovered a priori. It might seem that these two claims are inconsistent, if we suppose that in order to know something I have to know the features of reality that make it true. For if the procedure theory says that theorems are made true by laws, it would seem to follow that finding out that the theorems are true involves finding out the laws, so that mathematical practice ought to be inductive. But the phrase "make it true" is equivocal. If we use it within a linguistic practice, then what makes a true statement true is just the fact that it states. If we use it in semantic theory, then we mean what makes the linguistic practice we are describing one that can correctly be described as the stating of objective fact.

A semantic theory may be homophonic, in the sense that it explains a linguistic practice by using the very concepts that figure in the practice and which it seeks to explain. If it is nonhomophonic, then it uses concepts different from those used in the practice being explained. Platonism is a homophonic theory, for when it describes the relation between mathematical language and the world, it describes the world using matematical language. Thus according to Platonism, what makes statements about the number 6 objectively true or false are just the mathematical properties the number 6 objectively has. The procedure theory, on the other hand, is a nonhomophonic theory. When it describes the relation between mathematical language and the world, it uses a non-mathematical vocabulary and ap- 
peals instead to laws of nature. According to the procedure theory, what makes statements about the number 6 objectively true are the laws that underpin counterfactuals about the results of correct computation.

Because the procedure theory is a theory about the semantics of mathematics, it can say from outside that the practice depends on natural law without having to deny that knowledge within the practice is a priori. There is no contradiction in asserting both that a priori knowledge exists and that it is the laws of nature that make it possible. It remains to explain why a priori knowledge might depend on the laws of nature.

Counting involves going to an object and saying "one", going to the next and saying "two", never going to the same object twice, and stopping only when one has gone round all the objects. If I can identify all the objects the count will advance by exactly one for each and so will be determinate if I make no mistake. Hence provided I can correctly identify them, the objects are disposed to elicit from me a definite numeral. If I cannot identify them, still they would elicit the same numeral if I could identify them. Thus the number of a collection is determinate if each object is identifiable at least in principle. This establishes a priori that a count of identifiable objects is determinate. In the same way the proof of a proposition like $7+5=12$ establishes it a priori, since here too we appeal only to the possibility of correct identification.

Of course the argument would fail if the laws were not such as to allow events to be analyzed as the doings of identifiable continuants falling under sortals. That is why a priori knowledge can depend on laws. But if we had a prori reasons to think that any intelligible world must contain some suitable continuants, and therefore must have appropriate laws, then we would have an explanation consistent with the procedure theory of the $a$ priori nature of mathematics. Kant and Strawson ${ }^{22}$ have given

Strawson [1959] chapter 1. 
us such reasons, so that it seems plausible to think that the class of mathematically possible worlds coincides with the class of worlds that are intelligible. On this view, mathematical truths are not necessary since it is surely contingent that the world is intelligible. It is a consolation, however, that we can say a priori that any intelligible world is truly described by mathematics.

\section{REFERENCES}

Armstrong, D.M. [1983] What is a Law of Nature? (Cambridge: Cambridge University Press).

Ayer, A.J. [1967] Language, Truth and Logic (London: Gollancz). Berkeley, G. [1710] "Principles of Human Knowledge" in A New Theory of Vision and Other Writings (London: Everyman, 1963). Benacerraf, P. and H. Putnam, eds. [1983] Philosophy of Mathematics, Selected Readings, Second Edition, (Cambridge: Cambridge University Press).

Dummett, Michael [1973] Elements of Intuitionism (Oxford: Clarendon Press).

Field, Hartry [1989] Realism, Mathematics and Modality (Oxford: Basil Blackwell).

Frege, G. [1968] The Foundations of Arithmetic, trans. J.L. Austin, (Oxford: Basil Blackwell).

-[1891] Function and Concept in Frege: Translations, trans. M. Black and P. Geach (Oxford: Basil Blackwell, 1966).

Gödel, K. [1944] "Russell's Mathematical Logic" in Benacerraf and Putnam [1983].

- [1947] "What is Cantor's Continuum Problem" in Benacerraf and Putnam [1983].

Kant, I. [1933] Critique of Pure Reason, trans. N. Kemp Smith (Edinburgh: MacMillan).

Mill, J. Stuart [1967] A System of Logic (London: Longmans). Quine, W.V.O. [1936] "Truth by Convention" in The Ways of Paradox (Cambridge, Mass.: Harvard University Press, 1975).

Russell, B.A.W. [1956] An Essay on the Foundations of Geometry (New York: Dover).

Strawson, P.F. [1959] Individuals (London: Methuen). 
Tarski, A. [1956] The Concept of Logical Consequence in Logic, Semantics and Metamathematics, trans. J.H. Woodger (Oxford: Clarendon Press).

Wittgenstein, L. [1958] Philosophical Investigations (Oxford: Basil Blackwell).

- 1967] Remarks on the Foundations of Mathematics (Oxford: Basil Blackwell).

Wright, Crispin [1983] Frege's Conception of Numbers as Objects (Aberdeen: Aberdeen University Press). 


\section{RESUMEN}

Este artículo cuestiona las doctrinas usuales de que los términos matemáticos se refieren a objetos y de que las demostraciones matemáticas son deducciones lógicas. En su lugar, proponemos que captar un concepto matemático requiere típicamente dominar una técnica o un procedimiento asociado, y que diferentes demostraciones cuentan con que nosotros reflexionemos acerca de qué resultados tendrán los procedimientos pertinentes. Las demostraciones requieren mundos espacio-temporal-causales como el nuestro y fracasarían en mundos menos complacientes; esto implica que las verdades matemáticas no son necesarias ya que no se sostienen a través de todo el espacio lógico.

[Traducción de Ricardo Salles] 